\title{
Lyme disease: The bite of spring becomes the (diagnostic) menace of summer
}

With apologies to Igor Stravinsky, the "Rite of spring" in the United States has come to mean the "bite of spring." Specifically, that bite most often comes from the deer tick (Ixodes dammini), infected with the Lyme spirochete, Borrelia burgdorferi.

Since Allen C. Steere, MD, first described the disease in the Lyme area of Connecticut in 1975, 43 states have reported Lyme borreliosis to the Centers for Disease Control (CDC). The vast majority of these cases have occurred in New England, the mid-Atlantic states, and in the upper Midwest. In 1988, 4507 cases were reported to the $\mathrm{CDC}$, twice the number reported the previous year. A growing deer population, coupled with an encroachment of an increasing human population into the rural areas, has contributed to the continued increase in the number of exposures to the Lyme spirochete.

The deer tick produces nymphs that are active from late spring through early fall. Nymphs and adult ticks are found in tall grass, shrubs, and brush areas in a questing position, awaiting an unsuspecting host. Within a few days or several weeks after having been bitten by a tick, a person will develop erythema chronicum migrans (ECM). However, about $25 \%$ of patients develop no rash.

Other manifestations of Lyme disease have been categorized into three stages:

- Stage 1: Malaise, headache, fever, lymphadenopathy, ECM, arthralgia, and myalgia

- Stage 2: Recurrent ECM, carditis, aseptic meningitis, cranial neuritis, and radiculoneuritis

- Stage 3: Acrodermatitis chronica atrophicans, chronic neurologic syndrome, and chronic arthritis
However, Lyme disease remains a diagnostic challenge because the symptoms sometimes appear early, overlap, or do not manifest themselves at all. They may also mimic other forms of arthritis, rheumatism, and temporomandibular joint dysfunction.

Although the causative spirochete has been isolated from skin lesions, cerebrospinal fluid, and blood, serologic tests to detect the bacterium have yet to be standardized. The range of specificity and sensitivity varies considerably.

Drs Burdash and Fernandes review the diagnostic challenges of Lyme borreliosis beginning on page 573 in this issue. They focus on the current state of laboratory testing.

Because much is yet to be learned about Lyme disease, prevention remains the best "medicine" for avoiding this disease. That means wearing a long-sleeved, tightly knit shirt tucked inside pants. Pant legs should be tucked inside crew socks. Wearing a hat or cap keeps ticks out of hair and off of the scalp. Although not fool-proof, tick repellants containing permethrin or diethyltoluamide offer added protection.

Once attached to the victim's skin, the tick requires 24 hours to transmit the spirochete. Therefore, a tick inspection after a sojourn into grassy, wooded areas is an important preventive measure. Removing ticks carefully with a tweezer within this period affords protection.

The old adage, "An ounce of prevention is worth a pound of cure," proves more than appropriate in warding off the "bite of spring."

THOMAS WESLEY ALLEN, DO Editor in Chief 

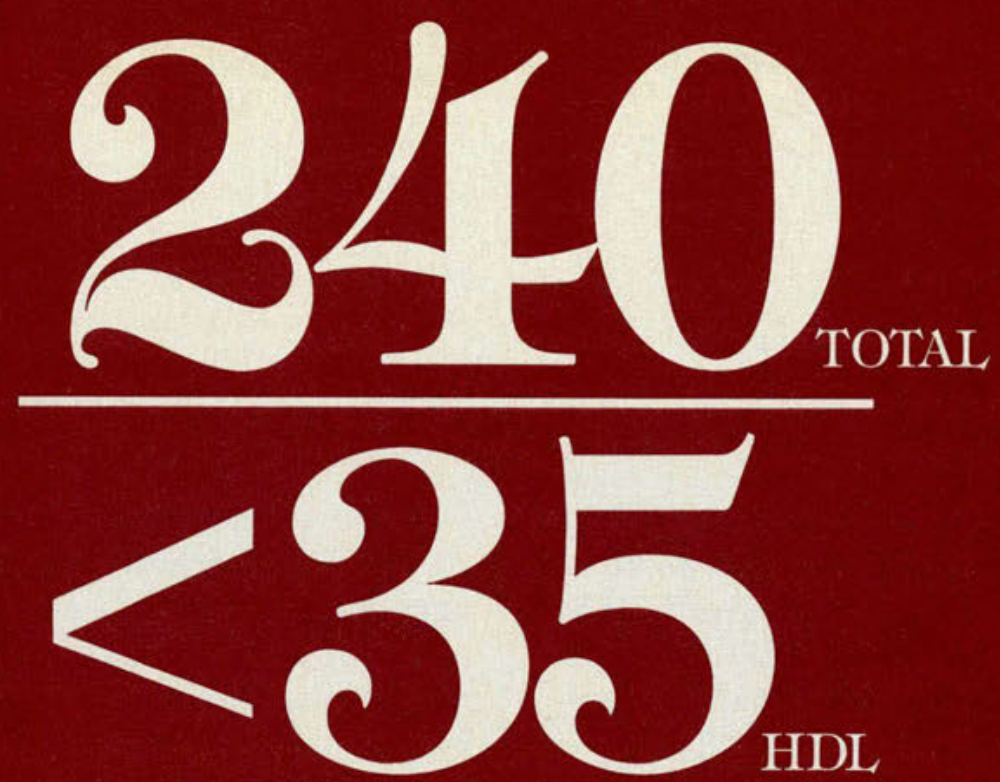

\section{Low HDL with elevated LDL and triglycerides: A common denominator of many heart attack victims}

Mixed hyperlipidemias - elevated cholesterol and triglycerides-are common among heart attack victims, and nearly two thirds of people who developed myocardial infarction in the PROCAM Trial had a low $(<35 \mathrm{mg} / \mathrm{dL})$ baseline level of HDL cholesterol. ${ }^{2}$ LOPID (gemfibrozil) is not indicated for the treatment of patients with low HDL cholesterol as their only lipid abnormality.

HEART ATTACK PATIENTS

$(\text { PROCAM TRIAL) })^{2}$

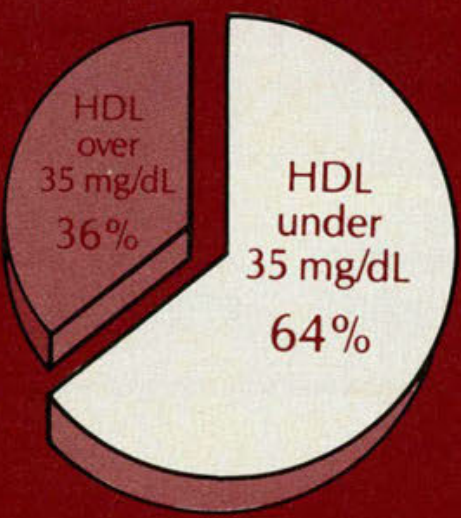




\section{A powerful case for (C) (gemfibrozil) $)_{\text {Tablets }}^{\text {600-mg }}$}

LOPID is indicated for reducing the risk of coronary heart disease in type Ilb patients with low HDL, in addition to elevated LDL and triglycerides, and who have had an inadequate response to weight loss, diet, exercise, and other pharmacologic agents such as bile acid sequestrants and nicotinic acid.

\section{Raised low HDL 25\%}

-in patients whose baseline $\mathrm{HDL}$ was $<35 \mathrm{mg} / \mathrm{dL}$ and median baseline LDL was $186 \mathrm{mg} / \mathrm{dL}$ in the landmark Helsinki Heart Study (HHS).

\section{Reduced heart attack incidence up to $62 \%$}

-in these HHS patients. ${ }^{3}$ Incidence of serious coronary events was similar for LOPID and placebo subgroups with baseline HDL above the median $(46.4 \mathrm{mg} / \mathrm{dL}){ }^{3}$

\section{RAISES HDL, LOWERS LDL AND TRIGLYCERIDES DRAMATICALLY REDUCES HEART ATTACK}

Contraindicated in patients with hepatic or severe renal dysfunction, including primary biliary cirrhosis, preexisting gallbladder disease, or hypersensitivity to gemfibrozil. LOPID may increase cholesterol secretion into the bile, leading to cholelithiasis. Caution should be exercised when anticoagulants are given in conjunction with LOPID.

\footnotetext{
- Defined as a combination of definite coronary death and/or definite myocardial infarction.
} $P=013 ; 95 \% \mathrm{Cl} 13.3$ to 111.5 .

References 1. Goldstein JL, Hazzard WR, Schrott HG, Bierman EL, Motulsky AG. Hyperlipidemia in coronary References 1. Goldstein IL, Hazzard WR, Schrott HG, Bierman EL, Motulsky AG. Hyperlipidemia in

2. Assmann G, Schulte H. PROCAM-Trial: Prospective Cardiovascular Münster Trial. Zurich: Panscientia Verlag: 2. Assmann G, Schulte H. PROCAM-Irial: Prospective Cardic
$1986: 8-9$ 3. Data on file, Medical Affairs Dept, Parke-Davis.

Please see last page of this advertisement for warnings, contraindications, and brief summary of prescribing information. 
Before prescribing, please see full prescribing information. A Brief Summary follows.

CONTRAINDICATIONS.

biliary cirrhosis.

2. Preexisting gallbladder disease (See WARNINGS)

3. Hypersensitivity to gemfibrozi

WARNINGS. 1. Because of chemical, pharmacological, and clinical similarities be. tween gemfibrozil and clofibrate, the adverse findings with clofibrate in two large clinical studies may also apply to gemfibrozil. In the first of those studies, the Coronary Drug Project, 1000 subjects with previous myocardial infarction were treated for five years with clofibrate. There was no difference in mortality between the clofibrate-treated sub jects and 3000 placebo-treated subjects, but twice as many clofibrate-treated subjects developed cholelithiasis and cholecystitis requiring surgery. In the other study, conducted by the World Health Organization (WHO), 5000 subjects without known coronary heart disease were treated with clofibrate for five years and followed one year beyond. There was a statistically significant, $29 \%$, higher total mortality in the clofibratetreated than in a comparable placebo-treated control group. The excess mortality was due to a $33 \%$ increase in noncardiovascular causes, including malignancy, postcholecystectomy complications, and pancreatitis. The higher risk of clofibrate-treated subjects for gallbladder disease was confirmed.

During the Helsinki Heart Study and in the $1 \frac{1}{2}$ year follow-up period since the trial was completed, mortality from any cause was $59(2.9 \%)$ in the Lopid group and 55 $(2.7 \%)$ in the placebo group. Mortality from any cause during the double-blind portion of the study was 44 deaths in the Lopid group and 43 in the placebo group. Because of the more limited size of the Helsinki Heart Study, this result is not statistically-

significantly different from the $29 \%$ excess mortality seen in the clofibrate group in the separate WHO study. Noncoronary heart disease related mortality showed a $58 \%$ greater trend in the Lopid group ( 43 vs 27 patients in the placebo group, $p=0.056$ ).

In the Helsinki Heart Study, the incidence of total malignancies discovered during the trial and in the $11 / 2$ years since the trial was completed was 39 in the Lopid group and 29 in the placebo group (difference not statistically significant). This includes 5 basal cell carcinomas in the Lopid group and none in the placebo group $(p=0.06$; historical data predicted an expected 4.7 cases in the placebo group). GI malignancies and deaths from malignancies were not statistically different between Lopid and placebo sub groups. Follow-up of the Helsinki Heart Study participants will provide further infor mation on cause-specific mortality and cancer morbidity.

2. A gallstone prevalence substudy of 450 Helsinki Heart Study participants showed trend toward a greater prevalence of gallstones during the study within the Lopid treatment group $7.5 \%$ vs $4.9 \%$ for the place bo group, a $55 \%$ excess for the gemfibrozil group). A trend toward a greater incidence of gallbladder surgery was observed for the Lopid group ( 17 vs 11 subjects, a $54 \%$ excess). This result did not differ statistically

from the increased incidence of cholecystectomy observed in the WHO study in the group treated with clofibrate. Both clofibrate and gemfibrozil may increase cholestero excretion into the bile leading to cholelithiasis. If cholelithiasis is suspected, gallbladder studies are indicated. Lopid therapy should be discontinued if gallstones are found 3. Since a reduction of mortality from coronary artery disease has not been demonstrated and because liver and interstitial cell testicular tumors were increased in rats, Lopid should be administered only to those patients described in the INDICATIONS AND USAGE section. If a significant serum lipid response is not obtained, Lopid should be discontinued

4. Concomitant Anticoagulants-Caution should be exercised when anticoagulants are given in conjunction with Lopid. The dosage of the anticoagulant should be reduced to maintain the prothrombin time at the desired level to prevent bleeding complications. Frequent prothrombin determinations are advisable until it has been definitely determined that the prothrombin level has stabilized.

5. Concomitant therapy with Lopid and Mevacors (lovastatin) has been associated with rhabdomyolysis, markedly elevated creatine kinase (CK) levels and myoglobinuria, leading in a high proportion of cases to acute renal failure. In most subjects who have had an unsatisfactory lipid response to either drug alone, the possible benefit of combined therapy with lovastatin and gemfibrozil does not outweigh the risks of severe myopathy rhabdomyolysis, and acute renal failure (See Drug Interactions). The use of fibrates alone, including Lopid, may occasionally be associated with myositis. Patients receiving Lopid and complaining of muscle pain, tenderness, or weakness should have prompt medical evaluation for myositis, including serum creatine kinase level determination. If myositis is suspected or diagnosed. Lopid therapy should be withdrawn.

6. Cataracts - Subcapsular bilateral cataracts occurred in 10\%, and unilateral in $6.3 \%$ of male rats treated with gemfibrozil at 10 times the human dose

PRECAUTIONS. 1. Initial Therapy - Laboratory studies should be done to ascertain that the lipid levels are consistently abnormal. Before instituting Lopid therapy, every attempt should be made to control serum lipids with appropriate diet, exercise, weight loss in obese patients, and control of any medical problems such as diabetes mellitus and hypothyroidism that are contributing to the lipid abnormalities

2. Continued Therapy-Periodic determination of serum lipids should be obtained and the drug withdrawn if lipid response is inadequate after 3 months of therapy.

3. Drug Interactions - (A) Lovastatin: Rhabdomyolysis has occurred with combined gemfibrozil and lovastatin therapy. It may be seen as early as 3 weeks after initiation of combined therapy or after several months. In most subjects who have had an unsatisfactory lipid response to either drug alone, the possible benefit of combined therapy with lovastatin and gemfibrozil does not outweigh the risks of severe myopathy, rhabdomyolysis, and acute renal failure. There is no assurance that periodic monitoring of

creatine kinase will prevent the occurrence of severe myopathy and kidney damage

(B) Anticoagulants: CAUTION SHOULD BE EXERCISED WHEN ANTICOAGU LANTS ARE GIVEN IN CONJUNCTION WITH LOPID. THE DOSAGE OF THE ANTI. COAGULANT SHOULD BE REDUCED TO MAINTAIN THE PROTHROMBIN TIME AT THE DESIRED LEVEL TO PREVENT BLEEDING COMPLICATIONS. FREQUENT PROTHROMBIN DETERMINATIONS ARE ADVISABLE UNTIL IT HAS BEEN

DEFINITELY DETERMINED THAT THE PROTHROMBIN LEVEL HAS STABILIZED.

4. Carcinogenesis, Mutagenesis, Impairment of Fertility-Long-term studies have been conducted in rats and mice at one and ten times the human dose. The inc dence of benign liver nodules and liver carcinomas was significantly increased in high dose male rats. The incidence of liver carcinomas increased also in low dose males, but this increase was not statistically significant $(p=0.1)$. In high dose female rats, there was a significant increase in the combined incidence of benign, and malignant liver shown to be carcinogenic with other fibrates.

Male rats had a dose-related and statisticall

tumors at 1 and 10 times the human dose

Electron microscopy studies have demonstrated a florid hepatic peroxisome prolifera tion following Lopid administration to the male rat. An adequate study to test for perox isome proliferation has not been done in humans but changes in peroxisome morphology have been observed. Peroxisome proliferation has been shown to occur in humans with either of two other drugs of the fibrate class when liver biopsies were com pared before and after treatment in the same individual

Administration of approximately three or ten times the human dose to male rats for 10 weeks resulted in a dose-related decrease of fertility. Subsequent studies demonstrated that this ted to the offspring

5. Pregnancy Category B-Reproduction studies have been performed in the rat at doses 3 and 9 times the human dose, and in the rabbit at 2 and 6.7 times the human dose. These studies have revealed no evidence of impaired fertility in females or harm to at the high dose levels. No significant malformations were found among almost 400 offspring from 36 litters of rats and 100 fetuses from 22 litters of rabbits.

There are no studies in pregnant women. In view of the fact that Lopid is tumorigenic in male and female rats, the use of Lopid in pregnancy should be reserved for those pa tients where the benefit clearly outweighs the possible risk to the patient or fetus.

6. Nursing Mothers - Because of the potential for tumorigenicity shown for gemfibrozil in rats, a decision should be made whether to discontinue nursing or discontinue the drug, taking into account the importance of the drug to the mother

Hematologic Changes - Mild hemoglobin, hematocrit and white blood cell decreases have been observed in occasional patients following initiation of Lopid therapy. However, these levels stabilize during long-term administration. Rarely, severe anemia, leukopenia, thrombocytopenia, and bone marrow hypoplasia have been reported. Therefore, periodic blood counts are recommended during the first 12 months of Lopid administration.

8. Liver Function - Abnormal liver function tests have been observed occasionally during Lopid administration, including elevations of AST (SGOT), ALT (SGPT), LDH, biliusually reversible when Lopid is discon. tinued. Therefore periodic liver function studies are recommended and Lopid therapy should be terminated if abnormalities persist.

9. Use in Children - Safety and efficacy in children have not been established. ADVERSE REACTIONS. In the double-blind controlled phase of the Helsinki Heart Study. 2046 patients received Lopid for up to 5 year In that study, the following adverse reactions were statistically more frequent in subjects in the Lopid group (placebo incidence in paren theses): gastrointestinal reactions, $34.2 \%$ (23.8\%); dyspepsia, 19.6\% (11.9\%); abdominal pain, $98 \%$ (5.6\%); acute appendicitis (histologically confirmed in most cases where data are available), $1.2 \%(0.6 \%)$; atrial fibrillation, $0.7 \%(0.1 \%)$

Adverse events reported by more than $1 \%$ of subjects, but without a significant difference between groups (placebo incidence in parentheses) were: diarrhea, 7.2\% (6.5\%); fatigue, $3.8 \%(3.5 \%)$; nausea/vomiting, $2.5 \%(2.1 \%)$; eczema, $1.9 \%(1.2 \%)$; rash, $1.7 \%$ $(1.3 \%)$; vertigo, $1.5 \%(1.3 \%)$; constipation, $1.4 \%(1.3 \%)$; headache, $1.2 \%(1.1 \%)$ Gallbladder surgery was performed in $0.9 \%$ of Lopid and $0.5 \%$ of placebo subjects, a $64 \%$ excess, which is not statistically different from the excess of gallbladder surgery observed in the clofibrate compared to the placebo group of the WHO study.

Nervous system and special senses adverse reactions were more common in the Lopid group. These included hypesthesia, paresthesias, and taste perversion. Other adverse reactions that were more common among Lopid treatment group subjects but where a causal relationship was not established include cataracts, peripheral vascular disease, and intracerebral hemorrhage.

From other studies it seems probable that Lopid is causally related to the cccurrence of musculoskeletal symptoms (See WARNINGS), and to abnormal liver function tests and hematologic changes (See PRECAUTIONS)

Reports of viral and bacterial infections (common cold, cough, urinary tract infections) were more common in gemfibrozil-treated patients in other controlled clinical trials of 805 patients. Additional adverse reactions that have been reported for gemfibrozil are listed below by system. These are categorized according to whether a causal relationship to treatment with Lopid is probable or not established:

CAUSAL RELATIONSHIP PROBABLE: Gastrointestinal: cholestatic jaundice; Central Nervous System: dizziness, somnolence, paresthesia, peripheral neuritis, decreased libido, depression, headache; Eye: blurred vision; Genitourinary: impotence; Musculoskeletal: myopathy, myasthenia, myalgia, painful extremities, arthralgia, synovitis, rhabdomyolysis (see WARNINGS and Drug Interactions under PRECAU TIONS); Clinical Laboratory: increased creatine phosphokinase, increased bilirubin, in creased liver transaminases (AST [SGOT], ALT [SGPT]), increased alkaline phosphatase Hematopoietic: anemia, leukopenia, bone marrow hypoplasia, eosinophilia; Im. munologic: angioedema, laryngeal edema, urticaria; Integumentary: exfoliative dermatitis, rash, dermatitis, pruritus.

CAUSAL RELATIONSHIP NOT ESTABLISHED: General: weight loss; Cardiac: extrasystoles; Gastrointestinal: pancreatitis, hepatoma, colitis; Central Nervous System: confusion, convulsions, syncope; Eye: retinal edema; Genitourinary: decreased male fertility Clinical Laboratory: positive antinuclear antibody; Hematopoietic: thrombocytopenia; Immunologic: anaphylaxis, Lupus-like syndrome, vasculitis; Integumentary: alopecia DOSAGE AND ADMINISTRATION. The recommended dose for adults is $1200 \mathrm{mg}$ administered in two divided doses 30 minutes before the morning and evening meal. MANAGEMENT OF OVERDOSE. While there has been no reported case of overdosage, symptomatic supportive measures should be taken should it occur tion trial with gemfibrozil in middle-aged men with dyslipidemia. N Eng/J Med 1987;317:1237-1245. 2. Manninen V. Elo O. Frick MH, et al: Lipid alterations and decline in the incidence of coronary heart disease in the Helsinki Heart Study. JAMA 1988 260:641-651. 3. Nikkila EA: Familial lipoprotein lipase deficiency and related disorders of chylomicron metabolism. In Stanbury J. B. et al. (eds.): The Metabolic Basis of Inherited Disease, 5th ed., McGraw-Hill, 1983, Chap. 30, pp. 622-642.

\section{PARKE-DAVIS}

Morris Plains, NJ 07950 USA 